\title{
Anxiety and health-related quality of life after cardiac surgery
}

Yalcin Guzelhan ${ }^{1}$, Murat Ugurlucan², Didem Melis Oztas³ ${ }^{3}$, Metin Onur Beyaz $^{2}$, Orcun Unal4, Nilufer Bektas ${ }^{5}$, Cenk Conkbayir ${ }^{6}$, Ufuk Alpagut ${ }^{7}$, Nilgun Bozbuga ${ }^{7}$

\author{
${ }^{1}$ Department of Psychiatry, Istanbul Education and Research Hospital, Istanbul, Turkey \\ 2Department of Cardiovascular Surgery, Medical Faculty, Istanbul Medipol University, \\ Istanbul, Turkey \\ ${ }^{3}$ Cardiovascular Surgery Clinic, Bagcilar Education and Research Hospital, Istanbul, \\ Turkey \\ ${ }^{4}$ Cardiovascular Surgery Clinic, Yedikule Chest Diseases and Thoracic Surgery \\ Education and Research Hospital, Istanbul, Turkey \\ ${ }^{5}$ Cardiovascular Surgery Clinic, Sisli Etfal Education and Research Hospital, Istanbul, \\ Turkey \\ ${ }^{6}$ Department of Cardiology, Near East University, Nicosia, Cyprus \\ ${ }^{7}$ Department of Cardiovascular Surgery, Istanbul Medical Faculty, Istanbul University, \\ Istanbul, Turkey
}

Submitted: 14 October 2019

Accepted: 3 November 2019

Arch Med Sci Atheroscler Dis 2020; 5: e27-e35

DOI: https://doi.org/10.5114/amsad.2020.94376

Copyright @ 2020 Termedia \& Banach

\section{Abstract}

Introduction: The aim of this study was to investigate the effect of elective cardiac surgery on health-related quality of life (HRQOL).

Material and methods: One hundred and eight (35 women and 73 men, mean age $62.3 \pm 12.7$ years) patients undergoing open heart surgery were enrolled in the study. Physical and mental domains of quality of life were measured using the 36-item Medical Outcomes Short-Form Health Survey (SF-36) self-administered questionnaire, and anxiety symptoms were assessed using the Spielberger State-Trait Anxiety Inventory (STAI). At baseline 108 patients filled out the SF-36 and STAI, and 102 patients at 6-month follow-up.

Results: It was found that there was significant improvement in three out of eight health domains: physical functioning $(p<0.02)$, role physical ( $p<$ $0.01)$, and social functioning $(p<0.04)$, at 6 -month follow-up. The two STAI sub-scores: the State Anxiety Inventory and the Trait Anxiety Inventory were found to be high $(\geq 40)$ both preoperatively and 6 months postoperatively. Postoperatively there was not any statistically significant decrease in the levels of anxiety.

Conclusions: This study suggests that the assessment of psychosocial factors, particularly the ongoing assessment of anxiety, could help in risk stratification and prediction of functional status and HRQoL in patients after cardiovascular surgery. Furthermore, the assessment of preoperative well-being should be integrated in routine care in order to identify and support patients with higher levels of anxiety.

Key words: health-related quality of life, anxiety, short-form health survey, state-trait anxiety inventory, risk factors, cardiac surgery.

\section{Introduction}

Open-heart surgeries such as coronary artery bypass grafting and valve replacements have been used to improve patient outcomes relat-

\author{
Corresponding author: \\ Yalcin Guzelhan MD \\ Department of Psychiatry \\ Istanbul Education \\ and Research Hospital \\ Kasap ilyas Mah \\ Org. Abdurrahman \\ Nafiz Gürman Cd., \\ 34098 Fatih/istanbul \\ Turkey \\ Phone: +90 5323854748 \\ E-mail: drguzelhan@gmail. \\ com
}


ed to cardiac symptoms, prolongation of life, and health-related quality of life [1-3]. Despite good results with low operative morbidity and mortality rates, these parameters alone do not give enough information on a patient's functional, emotional, and mental well-being. Recovery from cardiac surgery is not entirely determined by physical attributes and medical treatment, but social and psychological factors may also influence the process of postoperative and long-term recovery [4]. Postoperative health-related quality of life (HRQoL) is a primary goal of surgery in the elderly and an important aspect for many patients in their decision-making [1, 5].

Studies have suggested that the experience and recovery process after open-heart surgery may be more complex than anticipated and presents psychosocial and physical challenges that continue well after hospital discharge [6-10]. In particular, anxiety, depression, and self-rated health seem to influence the postoperative recovery and cardiac-specific quality of life after open heart surgery [11]. Reports on the benefits of cardiovascular surgery have focused on postoperative subjective health outcomes in terms of functional status, activity level, resumption of daily living activities, return to work, perceived psychological well-being, and self-reported health-related functional status as well as objective adverse cardiac events, and survival and complication rates [12, 13]. The World Health Organisation (WHO) [14] defined health as being "not only the absence of disease and infirmity but also the presence of physical, mental, and social well-being" [15]. The World Health Organisation Quality of Life (WHOQOL) Group describes quality of life as "an individual's perception of their position in life in the context of the culture and value system in which they live and in relation to their goals, expectations, and standards and concerns" [16].

Health-related quality of life encapsulates an individual's physical, emotional, and psychologic health, as well as social and functional status [17, 18]. Because HRQoL is not a tangible entity, a standardised method of measurement is required that is reliable, valid, responsive, and sensitive and that covers all health domains [19].

Questionnaires are the most popular method of measuring quality of life. The 36-item Medical Outcomes Short-Form Health Survey self-administered questionnaire (SF-36) (Medical Outcomes Trust, Boston, MA) is a multipurpose, short-form health survey with only 36 questions [20]. The SF-36 Health Survey is one of the most extensive standardised questionnaires developed to assess the functional status and well-being of patients and related to activities of daily living [21]. The SF-36 Health Survey which a eight-scale profile of scores is generic questionnaires for measuring both the physical and mental health of a patient. It is a generic measure, as opposed to one that targets a specific age, disease, or treatment group. Accordingly, the SF-36 has been useful in comparing general and specific populations, comparing the relative burden of diseases, differentiating the health benefits produced by a wide range of different treatments, and screening individual patients [22].

The Spielberger State-Trait Anxiety Inventory was designed to assess anxiety symptoms and was one of the first tests to examine state and trait anxiety at the same time $[23,24]$. The StateTrait Anxiety Inventory (STAI) consists of two 20item scales that measure "state" (current) and "trait" anxiety (general) (STAI-S and STAI-T). The STAI tests diagnose the level of anxiety and distinguish it from depressive syndromes [25].

The aim of the present study was to investigate the influence of psychological factors on cardiac surgery outcome and to evaluate the hypothesis that symptoms of anxiety are associated with adverse clinical outcomes and quality of life in patients who underwent elective open heart surgery.

\section{Material and methods}

The study population comprised 108 patients admitted for elective cardiovascular surgery. Inclusion criteria included adults older than 40 years, who were able to communicate responses to complete the questionnaires. Participants were scheduled to undergo elective open-heart surgery for the first time using a conventional sternotomy approach. Specific exclusion criteria included unstable angina, decompensated heart failure, life threatening arrhythmias, large left ventricular aneurysm, life-threatening cerebrovascular disease, severe renal or hepatic dysfunction, and major psychiatric disorders.

Demographic data on age, gender and education level (number of school years) along with patient-related factors: smoking, obesity, diabetes mellitus, hypertension, hyperlipidaemia, cerebrovascular disease, peripheral arterial disease, chronic renal failure, chronic obstructive pulmonary disease, previous myocardial infarction, and left ventricular dysfunction were recorded. At enrolment, participants reported their smoking history (grouped as never, former, < 20 cigarettes per day, and $\geq 20$ per day). To identify the risk factors (patient-related, cardiac, and operation-related) with their weights (additive percentage predicted mortality) the EuroSCORE model was used. According to the additive score, a low-risk group (EurOSCORE 1-2), a medium-risk group (EuroSCORE $3-5$ ), and a high-risk group (EuroSCORE $\geq 6$ ) were formed. 
The indicators of disease severity-specific variables related to the cardiovascular procedure such as total time on cardiopulmonary bypass, and aortic cross clamping were recorded. Postoperative complications (postoperative myocardial infarction, low cardiac output, congestive heart failure, cerebrovascular accident, severe arrhythmia, renal and pulmonary complications), and duration of intensive care unit and hospital stay and were recorded.

Follow-up visits of patients were scheduled 30 days, 3 months, and 6 months after the procedure. Before hospital discharge and at each follow-up visit, all patients underwent a physical examination. All those who participated in the study signed an informed consent form. The study was performed according to the principles of the Declaration of Helsinki.

\section{Definition of Health-Related Quality of Life}

The SF-36 consists of 36 questions in eight areas: general health perception (measurement of perceived overall health, including past and present health), physical functioning (indicates level of limitations in performance of daily living such as lifting, bending, kneeling, or walking moderate distance), bodily pain (represents the intensity, frequency, and duration of bodily pain and limitations in normal activities due to pain), mental health (measures the emotional, cognitive, and intellectual status of the patient), role-physical (measures the degree in performing of usual activities for age and social status), role-emotional (measures personal feeling of job performance at work or other activities), vitality (measures feeling of energy, fatigue, and tiredness), and social functioning (indicates the ability to develop and maintain mature social relationships). A score ranging from 0 to 100 is computed for the eight scales and the physical and mental summary scores, with higher values indicating a better health status $[20,26]$. The SF-36 can provide two summary measures: as a physical component score and a mental component score.

\section{Measurement of anxiety}

The two State-Trait Anxiety Inventory subscores - the state (STAI-S) and the trait (STAI-T) - have high concordance in people with diagnosed generalised anxiety disorder as well as in people without diagnosed anxiety. Representative items include statements such as "I feel nervous" and "I feel worried." These items are rated on a fourpoint scale of how well they describe the patient's current or typical mood from "not at all" to "very much so." STAI scores range from 20 to 80, with higher scores indicating more severe symptoms and greater anxiety. The two STAI sub-scores have high concordance in people with diagnosed generalized anxiety disorder as well as people without diagnosed anxiety. We accepted STAI scores $<40$ to indicate no or minimal symptoms and $\geq 40$ to indicate the presence of moderate or severe symptoms [25]. A range of psychosocial, clinical, and operative factors were collected as potential determinants of outcome.

\section{Statistical analysis}

Statistical analysis was performed using the statistical software SPSS 20.0 for Windows (SPSS Inc., Chicago, IL). Data are expressed as mean \pm standard deviation for continuous variables and as numbers with percentage for categorical variables. The discrete variables were compared using the $\chi^{2}$ test, and continuous variables were compared using a two-tailed $t$ test. Differences obtained from the pre- and postoperative assessments were compared by Wilcoxon matched-pairs rank-sum test. A $p$-value of 0.05 or less was considered to be significant.

\section{Results}

The study group consisted of one hundred eight patients, 35 women and 73 men, with a mean age of $62.3 \pm 12.7$ years (range: $40-81$ years). The mean number of years of education was $9.2 \pm 3.6$, (range: $0-19$ years). Reported smoking history was $80 \%$ of the patients. Preoperatively, $71.3 \%$ were in New York Heart Association class III-IV. Clinical risk factors and perioperative characteristics were assessed according to the additive risk score (EuroSCORE). The low-risk group $n=34$ (31.4\%), medium-risk group $n=48$ (44.4\%), and high-risk group $n=26(27.1 \%)$ were formed according to the EurOSCORE. Most of the patients were in lowand medium-risk groups, with a mean EuroSCORE of $4.2 \pm 2.7$ (range: $1-9$ ). The high cardiac operative risk group (EuroSCORE > 6) comprised $24 \%$ of the patients.

All of the patients underwent open-heart surgery (coronary artery bypass grafting, $n=69$ (63.9\%), coronary artery bypass grafting plus valve replacement, $n=9(8.3 \%)$, isolated valve replacement $n=30(27.8 \%))$ with cardiopulmonary bypass. There was no operative mortality. The morbidity rate (postoperative myocardial infarction in one, low cardiac output in two, severe arrhythmia in one, pulmonary complication in one) was $5 \%$ in 5 patients. There was no postoperative wound healing or instability of the bony thorax. Regarding to the mean intensive care and hospital stay, there were no statically significant differences between the groups of patients undergoing different types of cardiac procedures - coronary artery by- 
Table 1. Patients' characteristics

\begin{tabular}{|c|c|}
\hline Parameter & $N(\%)$ \\
\hline \multicolumn{2}{|l|}{ Sex: } \\
\hline Male & $73(67.6)$ \\
\hline Female & $35(32.4)$ \\
\hline \multicolumn{2}{|l|}{ Age [years]: } \\
\hline Mean (range) & $62.3 \pm 12.7(40-81)$ \\
\hline $40-50$ & $10(9)$ \\
\hline $51-60$ & $34(32)$ \\
\hline $61-70$ & $36(33)$ \\
\hline $71-80$ & $24(22)$ \\
\hline 81 and older & $4(4)$ \\
\hline \multicolumn{2}{|l|}{ Education level: } \\
\hline Mean (range) [years] & $9.2 \pm 3.6(0-19)$ \\
\hline Secondary school and lower & $84(78)$ \\
\hline High school & $17(16)$ \\
\hline University and higher & $7(6)$ \\
\hline \multicolumn{2}{|l|}{ Smoking history [cigarettes per day]: } \\
\hline Never & $19(18)$ \\
\hline Former & $3(3)$ \\
\hline$<20$ & $36(34)$ \\
\hline$\geq 20$ & $50(46)$ \\
\hline Hypertension & $42.1 \%$ \\
\hline Diabetes mellitus & $35.4 \%$ \\
\hline \multicolumn{2}{|l|}{ Functional class: } \\
\hline NYHA I & 0 \\
\hline NYHA II & $31(28.7)$ \\
\hline NYHA III & $65(60.2)$ \\
\hline NYHA IV & $12(11.1)$ \\
\hline \multicolumn{2}{|l|}{ Risk groups: } \\
\hline Mean (range) (EuroSCORE) & $4.2 \pm 2.7(1-9)$ \\
\hline Low & $34(32)$ \\
\hline Medium & $48(44)$ \\
\hline High & $26(24)$ \\
\hline \multicolumn{2}{|l|}{ Type of surgery: } \\
\hline CABG & $69(64)$ \\
\hline$C A B G+$ valve replacement & $9(8)$ \\
\hline Valve replacement & $30(28)$ \\
\hline Mean ACC time [min] & $43.2 \pm 13.3$ \\
\hline Mean CPB time [min] & $75.8 \pm 26.7$ \\
\hline Mean ICU time [days] & $2.2 \pm 0.5$ \\
\hline Mean hospitalisation time [days] & $7.6 \pm 1.6$ \\
\hline Morbidity & $5(5)$ \\
\hline
\end{tabular}

pass grafting or valve replacement. The patients' characteristics are summarised in Table I.

All of the 108 patients completed the shortform health survey (SF-36) and psychological self-reported questionnaires (STAI-S and STAI-T tests) preoperatively, and 102 of the study cohort (94.4\%) postoperatively. The repeated-measures analysis of the mean SF-36 and the mean STAI score at baseline and 6-month follow-up are summarised in Table II.

SF-36 scores did not show significant changes postoperatively in patients with low and medium cardiac operative risk according to the EuroSCORE. Statistically significant improvement of the scores was found in three out of eight health domains: physical functioning (48.8 \pm 25.4 vs. $59.8 \pm 22.3$ $p<0.02)$, role physical $(25.6 \pm 18.8$ vs. $41.8 \pm 38.8$ $p<0.01)$, and social functioning ( $57.8 \pm 23.8$ vs. 65.4 $\pm 25.2 p<0.04)$ at 6 -month follow-up. No significant changes were found for general health perception $(p=0.4)$, bodily pain $(p=0.3)$, mental health $(p=$ $0.1)$, role-emotional, and vitality $(p=0.1)$.

The high-risk group of patients were likely to have significant improvement in three of eight health domains following surgery than the lowand medium-risk group, according to the EuroSCORE $(p<0.05)$.

The both of the mean STAI sub-scores (STAI-S and STAI-T) were found to be high $(\geq 40)$ preoperatively. Of the 108 patients studied, $52 \%$ of the patient population ( 52 patients STAI scored $<40$ and 56 patients STAI scored $\geq 40$ ) were classified as presenting clinically significant anxiety symptoms.

The mean score on the preoperative STAI-S was $47.3 \pm 3.4$ points ( $<40$ points, mean $37.8 \pm 5.2$ and $\geq 40$ points, mean $57.8 \pm 8.1)$. The mean score on the preoperative STAI-T was $49.4 \pm 9.1$ points (scored < 40 points, mean $38.4 \pm 2.4$ and scored $\geq 40$ points, mean $61.8 \pm 7.5$ ).

The mean score at 6-month follow-up STAI-S was $41.9 \pm 6.2$ points, but it was not statistically significant $(p=0.1)$. Although it was observed that the mean STAI-S score was lower than any of the preoperative values, STAI-T scores remained relatively consistent compared with the preoperative value. The mean postoperative STAI-T score was $46.9 \pm 5.7$ points. There was no significant decreasing in the level of trait anxiety $(p>0.5)$. Almost half of the patients (46\%) still had borderline or abnormal STAI scores $(\geq 40)$ postoperatively.

There were some limitations of this study. One of the limitations is that the demographic variables may have had significant interactions with the variables HRQoL and anxiety but were not included in the regression analysis. Future studies should examine demographic variables and physical health status as predictors of benefits from cardiovascular surgery. 
Table II. Repeated-measures analysis of the mean SF-36 and the mean STAI score at baseline $(n=108)$ and 6-month $(n=102)$ follow-up

\begin{tabular}{|lccc|}
\hline Variable & $\begin{array}{c}\text { Baseline } \\
(n=108)\end{array}$ & $\begin{array}{c}6 \text {-month } \\
(n=102)\end{array}$ & $P$-value \\
\hline SF-36: & $50.1 \pm 13.8$ & $54.8 \pm 19.5$ & 0.4 \\
\hline General health & $48.8 \pm 25.4$ & $59.8 \pm 22.3$ & $<0.02$ \\
\hline Physical functioning & $48.9 \pm 33.4$ & $54.5 \pm 25.6$ & 0.3 \\
\hline Bodily pain & $36.4 \pm 27.3$ & $41.3 \pm 25.9$ & 0.1 \\
\hline Mental health & $25.6 \pm 18.8$ & $41.8 \pm 38.8$ & 0.1 \\
\hline Role physical & $48.4 \pm 37.4$ & $55.7 \pm 35.9$ & $<0.04$ \\
\hline Role emotional & $57.8 \pm 23.8$ & $65.4 \pm 25.2$ & 0.1 \\
\hline Social functioning & & $41.9 \pm 6.2$ & 0.5 \\
\hline STAI: & $47.3 \pm 3.4$ & $46.9 \pm 5.7$ & 0.1 \\
\hline STAI-S & $49.4 \pm 9.1$ & & \\
\hline STAI-T & & & \\
\hline
\end{tabular}

SF-36-Short-Form Health Survey, GH - general health, PF-physical functioning, BP-bodily pain, MH-mental health, RP-role physical, $R E$ - role emotional, VT - vitality, SF - social functioning, STAI - State-Trait Anxiety Inventory, STAI-S - State-Trait Anxiety Inventory "state" (current), STAI-T - State-Trait Anxiety Inventory "trait" (chronic).

\section{Discussion}

Due to advances in myocardial protection and surgical procedures, in-hospital mortality after open-heart surgery has decreased significantly. Also, important advances in perioperative anaesthesia and intensive care course have resulted in a substantial reduction in mortality during and after cardiac surgery. Because mortality has become relatively low after cardiac surgery, improvement in health status, changes in functioning status, and feeling of well-being have become important indicators of the result of cardiac surgery [3]. Improved quality of life has become one of the most important outcomes of cardiac surgery [5, 12]. Health-related quality of life can deteriorate postoperatively for several reasons: surgical complications, progression of non-cardiac diseases, neurological and psychological complications after cardiopulmonary bypass, etc. [11, 19, 26-28]. Therefore, postoperative quality of life after these operations has become an important measure of the effectiveness of cardiac surgery.

In cardiac pathology, disease-specific quality of life measures aim to accurately reflect a patient's experience of a specific illness or its treatment [29-31]. A common tool is the New York Heart Association (NYHA) classification. Generic health-related quality of life instruments are required to facilitate holistic and quantitative comparisons between different groups of patients. According to the WHO's disease-specific quality of life group, any generic health-related quality of life instrument should include physical, psychological, social, functional, and well-being domains [32].
The generic questionnaires most commonly used in diseases include: World Health Organisation Quality of Life Assessment Instrument (WHO$\mathrm{QOL})$, Nottingham Health Profile (NHP), EuroQuality of Life Questionnaire (EuroQoL, EQ-5D), and Medical Outcomes Study 36-item Short-Form Health Survey (SF-36).

The World Health Organisation quality of life questionnaire (WHOQOL) was developed as part of a multicentre international study in accordance with a standardised protocol, and it addresses six domains: physical health, level of independence, mental health, social relationships, spirituality/ religiousness/personal beliefs, and environment [32]. The WHOQOL-100 questionnaire consists of 100 questions and enables the evaluation of quality of life with regard to 28 subscales belonging to the aforementioned domains or pertaining to general quality of life and self-perceived health [33]. A shorter version of the instrument was developed (WHOQOL-Bref), consisting of 26 items and characterised by good psychometric properties; it evaluates four quality of life domains: physical health, psychological health, social relationships, and environment [34].

The Nottingham Health Profile (NHP) consists of two parts [35]. The first part includes 38 questions, while the second employs seven singular statements. The domains addressed include the physical, mental, and emotional spheres, as well as the respondent's social relationships. The NHP questionnaire contains 38 yes/no statements on health problems covering six dimensions of subjective health. The maximum score is 100 points; 
the higher the score, the more significant the health problem. The score does not take into account the respondents' good well-being, but only analyses the negative aspects of their functioning.

The Euro-Quality of Life Questionnaire (EuroQoL, EQ-5D) was developed for the evaluation of individuals aged 12 years or older [36]. The questionnaire analyses the respondent's health using five categories: self-care, mobility, usual daily living activities, level of pain and discomfort, and level of anxiety and depression. The answers pertain only to the current, where 100 denotes the best condition, and 0 the worst imaginable condition. This provides the questionnaire with great sensitivity to short-term changes, but the fluctuations of the results are significant, impeding the use of this tool [37].

A well-recognised HRQoL instrument is the Medical Outcomes Survey Short-Form 36 (SF-36) [20, 21]. The SF-36 questionnaire was created during the Medical Outcomes Study in order to evaluate the general health condition of various patient groups $[22,38]$. The so-called long Medical Outcomes Study version consisted of 149 questions grouped into 16 categories. Based on this version, the SF-36 questionnaire was developed. The SF-36 consists of 36 items grouped in eight general health parameters: general health perception, physical functioning, bodily pain, mental health, role-physical, role-emotional, vitality, and social functioning. The eight parameters can be grouped into two summarised scores: the physical health score (general health, physical functioning, bodily pain, role-physical) and the mental health score (mental health, role-emotional, vitality, social functioning). The number of possible responses per item varies from two to six. It is a self-administered instrument that takes about $15 \mathrm{~min}$ to complete. A Turkish version has been available since 1999 [26].

The most commonly used questionnaires specific to cardiovascular diseases include the following: the Minnesota Living with Heart Failure (MLHF) Questionnaire, Kansas City Cardiomyopathy Questionnaire (KCCQ), MacNew Heart Disease Health-Related Quality of Life Questionnaire (MacNew), Seattle Angina Questionnaire (SAQ), and the Atrial Fibrillation Severity Scale (AFSS).

The Minnesota Living with Heart Failure (MLHF) Questionnaire is used in the evaluation of chronic heart failure patients' related cardiac disease $[39,40]$. The questionnaire contains questions about the effects of heart failure on quality of life through 21 questions covering physical and emotional dimensions. The questionnaire is divided into three separate scales: physical, mental, and general. The answers are given on a scale from 0 (no influence) to 5 (strongest influence). After the score is totalled (0-105 points), the lower the score, the better the quality of life.
The Kansas City Cardiomyopathy Questionnaire (KCCQ) contains 23 items measuring self-reported health status in patients with heart failure [41]. Scales measured include: physical limitation, symptom stability, symptom frequency, symptom burden, total symptoms, self-efficacy, quality of life, social limitation, overall summary, and clinical summary.

The MacNew Heart Disease Health-Related Quality of Life Questionnaire (MacNew) was developed as a modification of the Quality of Life after Myocardial Infarction Questionnaire (QLMI) $[42,43]$. Its function is to evaluate patients after myocardial infarction or suffering from coronary artery disease or heart failure; it consists of 27 questions grouped into three domains: physical, mental, and social. The questionnaire evaluates the patient's symptoms and sense of wellbeing over the previous 2 weeks.

Seattle Angina Questionnaire (SAQ) evaluates the quality of life and functioning status of patients with coronary artery disease [44]. It consists of 19 questions grouped into five domains concerning: physical limitations, anginal frequency, anginal stability, treatment satisfaction, and disease perception from the past 4 weeks.

The Atrial Fibrillation Severity Scale (AFSS) is a disease-specific questionnaire for individuals with atrial fibrillation [45]. It consists of 14 questions pertaining to objective and subjective health changes. The questions focus mostly on the frequency, intensity, and duration of changes influenced by the disease.

The studies have demonstrated that one of the most appropriate methods to assess cardiac patients' HRQoL is the SF-36 survey $[1,2,18,22$, $28,38,46,47]$. Important predictors of improved quality of life outcomes have been shown to include age, gender, education, and comorbidities [48-50]. Preoperative mood and cognitive performance are also relevant predictors for early postoperative psychological dysfunctions, which, in turn, are associated with a prolonged hospitalisation and mortality after cardiac surgery [4, 11].

There is growing recognition of the importance of psychosocial factors in the recovery from medical and surgical procedures [51, 52]. Despite an increasing body of knowledge about neurocognitive changes after cardiac surgery, very little is known about possible neuropsychological consequences of severe stress reactions resulting from acute cardiac or pulmonary dysfunction and traumatic experiences, such as respiratory distress, severe pain, or anxiety, after surgery and during intensive care treatment [53]. Cardiac surgery causes, however, a considerable neurological and neurocognitive long-term morbidity [27]. Several studies have shown the importance of psychosocial factors in the development of coronary heart disease and/ 
or worsening of symptoms and survival in the presence of these factors $[4,46,47,54]$. The longterm negative influence of negative psychosocial stressors increases the severity of cardiovascular diseases [47]. The relationship between psychosocial factors and future cardiac events has been investigated mainly in population-based studies $[52,55]$. The studies reported a significant relationship between anxiety and cardiac death, and two of them also observed a dose-dependent relationship between the level of anxiety and the occurrence of fatal cardiac events. Recovery after open-heart surgery may be further complicated by preoperative and postoperative anxiety.

Anxiety was defined by Freud as "something felt", an emotional state that included feelings of apprehension, tension, nervousness, and worry accompanied by physiological arousal. In measuring anxiety, the importance of distinguishing between anxiety as an emotional state and individual differences in anxiety as a personality trait was emphasised [56]. Anxiety before cardiac surgery was accepted an an important factor for postoperative complications. In the early postoperative period, the relationship between anxiety and pain was reported in patients undergoing coronary surgery [11]. It has also been observed that patients with a high level of preoperative anxiety state and stronger anxiety trait respond worse to analgesic medication than patients with a low level of anxiety state and anxiety trait [57]. Among coronary heart disease patients undergoing coronary artery bypass grafting, anxiety was common and evaluated that it was associated with an increased mortality risk [58]. Although anxiety and depression were positively and highly correlated in these patients, only anxiety was found to be associated with increased mortality and morbidity [59].

The findings of the present study imply that neither age nor gender need be considered as risk factors for lower HRQoL when treatment decisions for cardiac surgery are made. Neither age nor sex predicted HRQoL, although fewer women than men ( 35 women vs. 73 men) had undergone open-heart surgery in the included studies. SF-36 scores did not show significant changes postoperatively in patients with low and medium cardiac operative risk. The high-risk group of patients were likely to have significant improvement in three of eight health domains following surgery than the low- and medium-risk groups, according to the EuroSCORE $(p<0.05)$. From this finding it was concluded that patients with high cardiac operative risk often have lower preoperative HRQoL and are more likely to have significant improvement postoperatively if their surgical outcome is satisfactory. Most of the patients included in the study were classified as presenting clinically sig- nificant anxiety symptoms. There was no significant decrease in the level of trait anxiety found postoperatively. The STAI scores of the female patients undergoing coronary surgery were reported to be significantly higher than men in state and trait anxiety both preoperatively and 6 months postoperatively. Postoperatively there was no significant decrease in the level of trait anxiety found when comparing the level of the state in female patients [60]. Female patients presenting for coronary surgery have different characteristics than male patients. Female patients tend to be older, less educated, and are more likely to be hypertensive, diabetic, and obese. In addition, women were more likely to be anxious and depressed compared with their male counterparts.

The results of the present study suggested that the assessment of anxiety using STAI could help in risk stratification. The selected interventions on mood and anxiety of patients preoperatively might have influenced the well-being after cardiac surgery.

In conclusion, this study suggests that the assessment of psychosocial factors, particularly the ongoing assessment of anxiety, could help in risk evaluation and prediction of functional status and health-related quality of life in patients after cardiovascular surgery. Measurement of HRQoL should be included in the routine assessment of patients in order to have a complete picture of their condition and augment conclusions about the effectiveness of treatment. Clinicians, patients, and relatives should be aware of this possibility, and an effort should be made for the preoperative identification of patients predisposed to severe chronic stress responses. Additionally, cardiovascular surgeons should pay attention to their patients' psychosocial functioning and, if necessary, advise them to seek professional guidance and support to maximise well-being.

In conclusion, patients' cardiac operative risk (EuroSCORE) as well as SF-36 and STAI scores should be assessed preoperatively. A larger and more homogeneous population of patients is needed to evaluate more precisely the influence of different psychosocial factors on long-term mortality and morbidity.

\section{Conflict of interest}

The authors declare no conflict of interest.

\section{References}

1. Shan L, Saxena A, McMahon R, Wilson A, Newcomb A. A systematic review on the quality of life benefits after aortic valve replacement in the elderly. J Thorac Cardiovasc Surg 2013; 145: 1173-89.

2. Jokinen JJ, Hippelainen MJ, Turpeinen AK, Pitkanen O, Hartikainen JE. Health related quality of life after coronary artery bypass grafting: a review of randomized controlled trials. J Card Surg 2010; 25: 309-17. 
3. Noyez L, de Jager MJ, Markou AL. Quality of life after cardiac surgery: under researched research. Interact Cardiovasc Thorac Surg 2011; 13: 511-4.

4. Blumenthal JA, Lett HS, Babyak MA, et al. NORG Investigators. Depression as a risk factor for mortality after coronary artery bypass surgery. Lancet 2003; 362: 604-9.

5. Abah U, Dunne M, Cook A, et al. Does quality of life improve in octogenarians following cardiac surgery? A systematic review. BMJ Open 2015; 5: e006904.

6. Puskas JD, Williams WH, Mahoney EM, et al. Off-pump vs conventional coronary artery bypass grafting: early and 1-year graft patency, cost, and quality-of-life outcomes: a randomized trial. JAMA 2004; 291: 1841-9.

7. Jensen $B \varnothing$, Hughes P, Rasmussen LS, Pedersen PU, Steinbruchel DA. Health-related quality of life following offpump versus on-pump coronary artery bypass grafting in elderly moderate to high-risk patients: a randomized trial. Eur J Cardiothorac Surg 2006; 30: 294-9.

8. Krannich JH, Lueger S, Weyers P, Elert O. Health-related quality of life two days before, ten days and one year after coronary artery bypass graft surgery. Thorac Cardiovasc Surg 2007; 55: 288-92.

9. Viswanathan GN, Mayurathan G, Hildreth T, Worthley SG, Zaman AG. Health related quality of life after percutaneous coronary revascularisation in patients with previous coronary artery bypass grafts: a two-year follow up study. Appl Res Qual Life 2011; 6: 311-24.

10. Cohen DJ, Van Hout B, Serruys PW, et al. Quality of life after $\mathrm{PCl}$ with drug-eluting stents or coronary-artery bypass surgery. N Engl J Med 2011; 364: 1016-26.

11. Middel B, El Baz N, Pedersen SS, van Dijk JP, Wynia K, Reijneveld SA. Decline in health-related quality of life 6 months after coronary artery bypass graft surgery: the influence of anxiety, depression, and personality traits. J Cardiovasc Nurs 2014; 29: 544-54.

12. Lie I, Arnesen H, Sandvik L, Hamilton G, Bunch EH. Health-related quality of life after coronary artery bypass grafting. The impact of a randomised controlled home based intervention program. Qual Life Res 2009; 18: 201-7.

13. Damgaard S, Lund JT, Lilleør NB, Perko MJ, Madsen JK, Steinbruchel DA. Comparably improved health-related quality of life after total arterial revascularization versus conventional coronary surgery Copenhagen arterial revascularization randomized patency and outcome trial. Eur J Cardiothorac Surg 2011; 39: 478-83.

14. WHO. Official records of the world health organization. In: WHO. 1948. Geneva.

15. Constitution of the World Health Organization. In: World Heath Organization. Handbook of Basic Documents. 5th ed. Geneva: Palais des Nations 1952: 3-52.

16. WHOQOL Group. Study Protocol for the World Health Organisation project to develop a quality of life assessment instrument (WHOQOL). Qual Life Res 1993; 2: 153-9.

17. Testa MA, Simonson DC. Assessment of quality-of-life outcomes. N Engl J Med 1996; 334: 835-40.

18. Wiebe S, Guyatt G, Weaver B, Matijevic S, Sidwell C. Comparative responsiveness of generic and specific quality-of life instruments. J Clin Epidemiol 2003; 56: 52-60.

19. Guyatt GH, Ferrans CE, Halyard MY, et al. Exploration of the value of health-related quality-of-life information from clinical research and into clinical practice. Mayo Clin Proc 2007; 82: 1229-39.

20. Ware JE, Kosinski M, Dewey JE. Changes between version 1 and version 2. W: How to score version 2 of the SF-
36 Health Survey (Standard and Acute forms). Ware JE, Kosinski M, Dewey JE (eds.). Quality Metric Incorporation, Lincoln 2002

21. Ware JE, Sherbourne CD. The MOS 36-item short form health survey (SF-36): I. Conceptual framework and item selection. Med Care 1992; 30: 473-83.

22. Kiebzak GM, Pierson LM, Campbell M, Cook JW. Use of the SF-36 general health status survey to document health-related quality of life in patients with coronary artery disease: effect of disease and response to coronary artery bypass graft surgery. Heart Lung 2002; 31: 207-13.

23. Speilberger CG, Lushene R. State-Trait Anxiety Inventory Manual. Palo Alto, CA: Consulting Psychologists Press, 1970.

24. Spielberger C. Manual for the State-Trait Anxiety Inventory (rev. ed.). Palo Alto (CA): Consulting Psychologists Press, 1983.

25. Spielberger CD, Sydeman SJ, Owen AE, Marsh BJ. Measuring Anxiety and Anger with the State-Trait Anxiety Inventory (STAI) and the State-Trait Anger Expression Inventory (STAXI). In: The Use of Psychological Testing for Treatment Planning and Outcomes Assessment. Maruish ME Mahwah NJ (eds.). US: Lawrence Erlbaum Associates Publishers 1999; 993-1021.

26. Aydemir Ö. Konsültasyon-Liyezon psikiyatrisinde yaşam kalitesi ölçümü: Kısa Form-36 (SF-36). Psikiyatri Psikoloji Psikofarmakoloji Dergisi 1999.

27. Newman MF, Grocott HP, Mathew JP, et al. Report of the substudy assessing the impact of neurocognitive function on quality of life 5 years after cardiac surgery. Stroke 2001; 32: 2874-81.

28. Colak Z, Segotic I, Uzun S, Mazar M, Ivancan V, MajericKogler V. Health related quality of life following cardiac surgery correlation with EuroSCORE. Eur J Cardiothorac Surg 2008; 33: 72-6.

29. de Quadros AS, Lima TC, Rodrigues AP, et al. Quality of life and health status after percutaneous coronary intervention in stable angina patients: results from the real-world practice. Catheter Cardiovasc Interv 2011; 77: 954-60.

30. Li R, Yan BP, Dong M, et al. Quality of life after percutaneous coronary intervention in the elderly with acute coronary syndrome. Int J Cardiol 2012; 155: 90-6.

31. Soo Hoo SY, Gallagher R, Elliott D. Systematic review of health-related quality of life in older people following percutaneous coronary intervention. Nurs Health Sci 2014; 16: 415-27.

32. The WHOQOL Group. The Development of the World Health Organization Quality of Life Assessment Instrument (the WHOQOL). In: Quality of Life Assessment: International Perspectives. Orley J, Kuyken W (eds.). Springer, Berlin, Heidelberg 1994.

33. The World Health Organization Quality of Life assessment (WHOQOL): position paper from the World Health Organization. Soc Sci Med 1995; 41: 1403-9.

34. Szabo S. The World Health Organization Quality of Life (WHOQOL) assessment instrument. In: Quality of life and pharmacoeconomics in clinical trials. Spilker B (ed.). Lippincott-Raven Publishers, Philadelphia 1996; 355-62.

35. Prieto L, Alonso J, Ferrer M, Anto JM. Are results of the SF-36 health survey and the Nottingham Health Profile similar? A comparison in COPD patients. Quality of Life in COPD Study Group. J Clin Epidemiol 1997; 50: 463-73.

36. Lukkarinen $\mathrm{H}$, Hentinen $M$. Assessment of quality of life with the Nottingham Health Profile among patients with coronary heart disease. J Adv Nurs 1997; 26: 73-84. 
37. EuroOol - a new facility for the measurement of health-related quality of life. The EuroQol Group. Health Policy 1990; 16: 199-208.

38. Failde I, Ramos I. Validity and reliability of the SF-36 Health Survey Questionnaire in patients with coronary artery disease. J Clin Epidemiol 2000; 53: 359-65.

39. Riegel B, Moser DK, Glaser D, et al. The Minnesota Living With Heart Failure Questionnaire: sensitivity to differences and responsiveness to intervention intensity in a clinical population. Nurs Res 2002; 51: 209-18.

40. Supino PG, Borer JS, Franciosa JA, et al. Acceptability and psychometric properties of the Minnesota Living With Heart Failure Questionnaire among patients undergoing heart valve surgery: validation and comparison with SF-36. J Card Fail 2009; 15: 267-77.

41. Green CP, Porter CB, Bresnahan DR, Spertus JA. Devel opment and evaluation of the Kansas City Cardiomyopathy Questionnaire: a new health status measure for heart failure. J Am Coll Cardiol 2000; 35: 1245-55.

42. Dixon T, Lim L, Oldridge NB. The MacNew health-related quality of life instrument: reference data for users. Qual Life Research 2002; 11: 173-83.

43. Hofer S, Lim LL, Guyatt G, Oldridge N. The MacNew Heart Disease Health-Related Quality of Life instrument: a summary. Health Qual of Life Outcomes 2004; 2: 3.

44. Spertus JA, Winder JA, Dewhurst TA, et aL. Development and evaluation of the Seattle Angina Questionnaire: A new functional status measure for coronary artery disease. J Am Coll Cardiol 1995; 25: 333-41.

45. Dorian P, Mangat I. Quality of life variables in the selection of rate versus rhythm control in patients with atria fibrillation: observations from the Canadian Trial of Atrial Fibrillation. Card Electrophysiol Rev 2003; 7: 276-9.

46. Calvert MJ, Freemantle N, Cleland JG. The impact of chronic heart failure on health-related quality of life data acquired in the baseline phase of the CARE-HF study. Eur J Heart Fail 2005; 7: 243-51.

47. Roberge M, Dupuis G, Marchland A. Post-traumatic stress disorder following myocardial infarction: prevalence and risk factors. Can J Cardiol 2010; 26: e170-5.

48. Gjeilo KH, Wahba A, Klepstad P, Lydersen S, Stenseth R. The role of sex in health-related quality of life after cardiac surgery: a prospective study. Eur J Cardiovasc Prev Rehabil 2008; 15: 448-52.

49. Martin LM, Holmes SD, Henry LL, et al. Health-related quality of life after coronary artery bypass grafting surgery and the role of gender. Cardiovasc Revasc Med 2012; 13: 321-7.

50. Peric V, Borzanovic M, Stolic R, et al. Quality of life in patients related to gender differences before and after coronary artery bypass surgery. Interact Cardiovasc Thorac Surg 2010; 10: 232-8.

51. Skodova Z, van Dijk JP, Nagyova I, et al. Psychosocial predictors of change in quality of life in patients after coronary interventions. Heart Lung 2011; 40: 331-9.

52. Székely A, Balog P, Benkö E, et al. Anxiety predicts mortality and morbidity after coronary artery and valve surgery. A 4-year follow-up study. Psychosom Med 2007; 69: 625-31.

53. Graf J, Koch M, Dujardin R, Kersten A, Janssens $U$. Health-related quality of life before, 1 month after, and 9 months after intensive care in medical cardiovascular and pulmonary patients. Crit Care Med 2003; 31 2163-9.

54. Rosenbloom J, Wellenius GA, Mukamal KJ, Mittleman MA. Self-reported anxiety and the risk of clinical events and atherosclerotic progression among patients with Coronary Artery Bypass Grafts (CABG). Am J Cardiol 2009; 158: 867-73.

55. Grös, DF, Antony MM, Simms LJ, McCabe RE. Psychometric properties of the State-Trait Inventory for Cognitive and Somatic Anxiety (STICSA): comparison to the StateTrait Anxiety Inventory (STAI). Psychol Assess 2007; 19: 369-81.

56. De Jong MJ, Hall LA. Measurement of anxiety for patients with cardiac disease: a critical review and analysis. J Cardiovasc Nurs 2006; 21: 412-9.

57. Greszta E, Siemińska MJ. Relationship of preoperative anxiety-state and anxiety-trait in patients qualified for coronary artery bypass graft surgery to the perception of postoperative pain and other pain complaints. Ann Acad Med Stetin 2008; 54: 157-63.

58. Rymaszewska J, Kiejna A, Hadrys T. Depression and anxiety in coronary artery bypass grafting patients. Eur Psychiatry 2003; 18: 155-60.

59. Wellenius GA, Mukamal KJ, Kulshreshtha A, et al. Depressive symptoms and the risk of atherosclerotic progression among patients with coronary artery bypass grafts. Circulation 2008; 117: 2313-9.

60. Güzelhan Y, Conkbayır C, Uğurlucan M, Yıldız CE, Alpagut $U$, Bozbuğa N. Gender differences in patients with anxiety after coronary artery bypass surgery. Heart Surg Forum 2018; 21: e165-9. 